\title{
Analysis of Grey Level Co-Occurrence Matrix Algorithm Features Using QR Image
}

\author{
R Sandha ${ }^{1}$ and M Ganaga Durga ${ }^{2}$ \\ ${ }^{1}$ Mannar Thirumalai Naicker College \\ ${ }^{2}$ Sri Meenakshi Government College for Women
}

May 5, 2020

\begin{abstract}
Key generation for data security is an important feature in cloud environment. Designing key from extracted feature is used to overcome the problem of vulnerable and mathematical attack. Our proposed work is concentrate on some of the features of Grey Level Co -occurrence Matrix(GLCM) co-relation, entropy, Angular second Moment(ASM) and Inverse Differential Moment(IDM) .Using these features Comparative analysis on GLCM Algorithm features of Quick Response (QR)image is made with existing different image such as iris , cartoon and Magnetic Resonance Imaging (MRI) image features produced by GLCM. We came to know that GLCM can extract the feature under single direction and single scale.
\end{abstract}

\section{Hosted file}

Analysis Of Grey Level Co (1) (1).docx available at https://authorea.com/users/293936/ articles/422083-analysis-of-grey-level-co-occurrence-matrix-algorithm-features-using-qrimage 